\title{
Acoplamiento del producto interno bruto a la emisión de gases de efecto invernadero: un limitante para lograr crecimiento verde en Costa Rica
}

\section{Gross domestic production it is coupling with the green house gas emission in Costa Rica, a limitiation to move into green growing}

M.Sc. Manuel Campos-Rudin

Universidad de Costa Rica, Costa Rica

mcamposrudin@gmail.com

\section{RESUMEN:}

Se analizó, por medio de indicadores económicos y ambientales, si el modelo de crecimiento económico de Costa Rica está o no acoplado a la emisión de gases de efecto invernadero. Para ello se recolectó información de bases de datos abiertas para los años 1990 a 2014. Los resultados mostraron, bajo un modelo de regresión logística, que el crecimiento del producto interno bruto y el uso de energía en kilogramos equivalentes de petróleo son variables predictoras significativas para la variable dependiente de emisión total de gases efecto invernadero. En un análisis puntual de 2008 a 2014, se observa un desacoplamiento, pero estos deben ser analizados hasta el 2018 con un nuevo inventario nacional de gases de efecto invernadero. Se concluyó que Costa Rica, de forma histórica, posee un crecimiento acoplado a la emisión total de gases de efecto invernadero, lo cual limita un modelo de crecimiento verde.

\section{ABSTRACT:}

By means of economic and environmental indicators, an analysis was made as to whether Costa Rica's economic growth model is or not linked to greenhouse gas emissions. To this effect open database information was collected for the years between 1990 and 2014 . According to a logistic regression model, the results revealed that the growth of the gross domestic product and the use of energy in kilogram oil equivalent are significant predictor variables for the variable dependent on the total emission of greenhouse gases. In an occasional analysis from 2008 to 2014, a disconnection was observed, but the analysis must continue until 2018 based on a new national inventory of greenhouse gases. It was concluded that Costa Rica's growth is historically linked to the total emission of greenhouse gases, which limits a model of green growth.

\section{RESUMO:}

Analisou-se, através de indicadores econômicos e ambientais, se o modelo de crescimento econômico da Costa Rica está ou não acoplado à emissão de gases de efeito estufa. Para isso, foram coletadas informações de bancos de dados abertos para os anos de 1990 a 2014. Os resultados mostraram, sob um modelo de regressão logística, que o crescimento do produto interno bruto e o uso de energia em quilogramas equivalentes de petróleo são variáveis preditoras significativas para a variável dependente da emissão total de gases de efeito estufa. Em uma análise pontual de 2008 a 2014, observa-se um desacoplamento, mas estes devem ser analisados até $2018 \mathrm{com}$ um novo inventário nacional de gases de efeito estufa. Concluiu-se que a Costa Rica, historicamente, possui um crescimento acoplado à emissão total de gases de efeito estufa, o qual limita um modelo de crescimento verde.

\section{RÉSUMÉ:}

Au moyen d'indicateurs économiques et environnementaux, une analyse a été faite pour déterminer si le modèle de croissance économique du Costa Rica est ou non lié à l'émission de gaz à effet de serre. A cet effet, l'information de bases de données ouvertes a été rassemblée pour les années de 1990 à 2014. Les résultats ont révélé, suivant un modèle de régression logistique, que la croissance du produit intérieur brut et l'utilisation d'énergie en kilogrammes équivalent pétrole sont des variables de prédiction pour la variable dépendante de l'émission totale de gaz à effet de serre. Dans une analyse ponctuelle de 2008 à 2014 , une déconnexion est observée mais il faut poursuivre l'analyse jusqu'en 2018 sur base d'un nouvel inventaire national de gaz à effet de serre. Il a été conclu que la croissance du Costa Rica est historiquement liée à l'émission totale de gaz à effet de serre, ce qui limite un modèle de croissance verte.

\section{PALABRAS CLAVES \\ CRECIMIENTO \\ SOSTENIBLE, \\ CRECIMIENTO \\ ECONÓMICO, CAMBIO CLIMÁTICO}

\author{
KEYWORDS \\ SUSTAINABLE \\ GROWTH, \\ ECONOMIC \\ GROWTH, CLIMATE \\ CHANGE
}

PALAVRAS CHAVES
CRESCIMENTO
SUSTENTÁVEL,
CRESCIMENTO
ECONÔMICO,
MUDANÇAS CLIMÁTICAS

MOTS CLÉS

CROISSANCE DURABLE, CROISSANCE ECONOMIQUE, CHANGEMENT CLIMATIQUE 


\section{INTRODUCCIÓN}

El planeta tierra sufre un proceso de calentamiento global denominado como Cambio climático (CC); este proceso incrementa la temperatura y ha sido asociado a nuestros modelos de crecimiento económico, los cuales llevan a impactos directos en las economías de los países, de los cuales los pobres son los que sufrirán mayores daños económicos, sociales y ambientales (Moore y Diaz, 2015). Los procesos generadores del cambio climático tienen en gran medida origen antropocéntrico y han sido asociados con el modelo de crecimiento (Greiner, 2004). El consumo y las demandas de la población mundial son crecientes para el planeta; en los últimos cien años, la población mundial se triplicó y se espera un incremento del 30 \% en los próximos treinta y cinco años. El consumo de la población para satisfacer sus necesidades lleva a una presión de los sistemas ecológicos y la respuesta puede localizarse en un consumo más eficiente (Millenium Ecosystem Assestent, 2005). También se debe pensar en el desarrollo de procesos de producción más limpios y rentables (Martínez \& Porcelli, 2017). Se deben gestar procesos que generen un desarrollo sostenible, que involucre los ejes sociales, económicos y ambientales.

Los conceptos de crecimiento verde (CV o "Green Growth") fueron planteados en la 5ta Conferencia Ministerial sobre Ambiente y Desarrollo, en marzo y junio de 2005 en Seúl, Corea. En 2009, treinta y cuatro países firmaron un convenio para generar y potenciar el crecimiento verde bajo el impulso de la Organización para Cooperación y Desarrollo Económico (OECD, por sus siglas en inglés). El CV se define de la siguiente forma:

Crecimiento verde significa fomentar el crecimiento y el desarrollo económicos y al mismo tiempo asegurar que los bienes naturales continúen proporcionando los recursos y los servicios ambientales de los cuales depende nuestro bienestar. Para lograrlo, debe catalizar el desarrollo sostenible, inversión e innovación que apuntalen el crecimiento sostenido y abran paso a nuevas oportunidades económicas (OECD, 2011, p 17).

Esta visión pretende armonizar el crecimiento económico con la sostenibilidad ambiental; también pretende fomentar un uso eficiente de los ecosistemas, donde surjan sinergias positivas entre el medio ambiente y la economía de los países (Allen y Clouth, 2012).

Otra definición fue propuesta por The Global Green Growth Institute (GGGI), la cual toma en cuenta, además de los aspectos ecológicos y económicos, la inclusión social y reducción de la pobreza:

El crecimiento verde es el desarrollo de un modelo que busca desarrollar el crecimiento económico, el cual incluye un desarrollo ambientalmente sostenible y socialmente inclusivo (GGGI, 2015, p12).

En el último reporte de la OECD (2017) Green Growth Indicators, se muestra que persiste el aumento de emisiones de GEI acoplados al crecimiento económico PIB de las naciones vinculadas a la OECD (Costa Rica fue analizada como país no miembro). El reporte indica que Costa Rica se halla ubicada en la zona de naciones con un desacoplamiento parcial de su crecimiento económico de las emisiones totales de GEI equivalentes $\mathrm{CO} 2$. En Costa Rica, se observa un fuerte incremento del PIB basado en emisiones de $\mathrm{CO} 2$ de manera continua desde 1995 hasta 2014 y ocupa la tercera posición en el análisis realizado en su indicador "producción basado en la producitividad-CO2", el cual se calcula usando el PIB real generado por unidad de $\mathrm{CO} 2$ emitido (USD/CO2 kg) (OECD, 2017). 
En Costa Rica, el Ministerio de Ambiente y Energía (Minae) tiene una propuesta en análisis para gestar las políticas nacionales de consumo y producción sostenible para el período 2018-2030. En este documento, se ponen de manifiesto los ejes estratégicos para enlazar una gestión ambiental con los retos de los objetivos de desarrollo del siglo XXI (ODS) en los diferentes sectores, comercio, agricultura, industria, entre otros, así como la educación y sus retos para su implementación en Costa Rica (Minae, 2017). Los ejes estratégicos de esta propuesta son la producción sostenible (no alimentaria), sistemas agroalimentarios sostenibles, turismo sostenible, estilos de vida sostenibles, construcción sostenible, compras públicas sostenibles y fortalecimiento institucional.

La implementación de estos ejes, más otros que el país posee (por ejemplo, los pagos por servicios ambientales), permitirán que Costa Rica se dirija hacia el CV, pero debe lograrse una reducción en la emisión los gases de efecto invernadero (GEI) o crear una senda hacia una economía baja en emisiones de carbono, también conocida como descarbonización. Costa Rica, en su inventario 2012 de GEI, tuvo una emisión total de 11250,50 Gg en CO2 equivalentes, de los cuales $7213,83 \mathrm{Gg}$ en $\mathrm{CO} 2$ equivalentes son del sector energía. En Costa Rica, el 64,12 \% de las emisiones de GEI provienen del sector energía (este incluye transportes, producción de energía eléctrica, comercio, industria y otras), donde el subsector transportes emite el 68,7 \%, el subsector de la industria de manufactura y construcción el 15,6 \% y el subsector de la generación de energía el 8,25\%. Todo estos representan el 92,55 \% del total de emisiones GEI del sector energético (Chacón, Jiménez, Montenegro, Sasa y Blanco, 2012).

Costa Rica ha sido un país que impulsa y promueve una agenda ambiental a nivel mundial; esto quedó demostrado en el Acuerdo de París para el cambio climático en 2015 (Comisión Europea - Acción por el Clima, 2015) y al adoptar como un compromiso los objetivos de desarrollo sostenible (ODS) planteados por la ONU (Organización de las Naciones Unidas, 2015). Además, busca posicionarse como un país que promueve la conservación de los recursos biológicos. De manera reciente, el Gobierno actual de Costa Rica ha lanzado un plan de descarbonización, el cual tiene varios ejes, entre los cuales están el uso de energías limpias, eliminar dependencia de hidrocarburos fósiles, conservación de recursos naturales, infraestructura verde y transporte público, entre otros lineamientos (Minae, 2018). La toma de decisiones para reducir la emisión de GEI, descarbonizar la economía y lograr un desacoplamiento del producto interno bruto (PIB) de las emisiones totales de GEI, pueden gestarse evaluando indicadores ambientales e indicadores económicos que expliquen o puedan predecir este acoplamiento, además de servir como indicadores que muestren el éxito o fracaso de reformas. Sin el análisis mediante indicadores ambientales y económicos en forma conjunta, los resultados de las políticas y acciones gestadas no serían debidamente validadas; esto justifica el usar modelos multivariados con una herramienta estadística sólida para obtener ecuaciones que nos expliquen cuáles son las variables que indiquen en forma significativa los resultados que se generen.

El presente trabajo tuvo como objetivo analizar la situación de Costa Rica en cuanto a sus indicadores ambientales y económicos, para comprender si el crecimiento de su economía estaba o no vinculada con la emisión de gases de efecto invernadero (GEI), donde el análisis de la gestión de la energía juega un papel determinante. Se tiene como hipótesis del estudio que Costa Rica, al declarar metas de carbono neutralidad, desarrollo de pagos por servicios ambientales, existencia de líneas de crédito para proyectos verdes y su fuerte proyección hacia la conservación, su crecimiento económico debería mostrar un desacoplamiento del PIB de las emisiones de gases de efecto invernadero. Para lograr estos objetivos, se obtuvo la información de bases de datos abierta para lograr confrontar los resultados obtenidos con los emitidos por el informe de la OECD de 2017 en temas de indicadores ambientales. 


\section{METODOLOGÍA}

\section{Tipo de estudio}

Se realizó un estudio bajo el paradigma cuantitativo multivariado retrospectivo al realizar los análisis de un grupo de indicadores ambientales y económicos extraídos de bases de datos abiertas, donde fueron analizados los años desde 1990 hasta 2014 (ver tabla 1). El análisis se concluye en 2014, pues es el último año con información completa para todos los indicadores de análisis.

$\begin{aligned} & \text { Indicadores ambientales, energéticos y económicos obtenidos de } \\ & \text { las bases de datos abiertas consultadas. }\end{aligned}$
$\begin{aligned} & \text { Indicadores ambientales - } \\ & \text { energéticos }\end{aligned}$

Tabla 1. Indicadores ambientales, energéticos y económicos obtenidos de las bases de datos abiertas consultadas. Fuente: Elaboración propia.

Los años analizados o seleccionados para el estudio tienen como justificante varios eventos relevantes en temas ambientales en Costa Rica: los pagos de servicios de ambientales iniciaron en el año 1997, el país propuso la carbono neutralidad en 2008 y para el año 2012 se presentó su último inventario país de gases de efecto invernadero. Estos años permiten brindar un análisis integral con datos publicados en bases abiertas, sobre si los resultados fueron concordantes con las grandes propuestas del país, estos datos se presentan antes de la de la posible publicación del inventario nacional de GEI para Costa Rica para el año 2015 y también antes de que iniciara el Plan para la Descarbonización Nacional 2018.

Para explicar la compleja relación del crecimiento económico y su vinculación con variables ambientales y energéticas, donde variables independientes pueden estar vinculadas a una variable dependiente, es necesario realizar modelos de regresión múltiple. Una bondad de este tipo de análisis es que las diversas variables predictoras pueden ser continuas o dicotómicas. También existen diferentes modelos con pasos para analizar las diversas variables para obtener la mejor ecuación que explique el modelo. Bajo la regresión multivariada, en este estudio se obtendrá la mejor ecuación que explique los resultados del modelo de construcción tipo stepwise o paso a paso, donde las variables predictoras son introducidas una a la vez para determinar cuáles de las variables generan el mejor modelo posible; si una variable no es significativa, es removida del proceso (Boslaugh, 2013). 


\section{Recolección de los datos ambientales, de GEI y económicos utilizados para Costa Rica}

Los datos se obtuvieron de bases datos abiertas de los siguientes organismos: Banco Mundial (2017) y Centro de Investigación Climatológica (CIAT, por sus siglas en inglés) (World Resources Institute, 2017). Los datos obtenidos de estas bases de datos fueron compilados en hojas de cálculo para su posterior análisis. Todas las variables seleccionadas para el estudio estaban disponibles para Costa Rica desde 1990 hasta 2014.

\section{Análisis estadístico de los datos}

La estadística descriptiva básica, la generación de las ecuaciones y la R2 para cada una de las variables económicas y ambientales para Costa Rica se realizaron con el programa Excel. Con estos análisis, se buscó conocer si los indicadores económicos, ambientales y vinculados a energía tenían tendencias lineales y si las pendientes de las ecuaciones de mejor ajuste eran positivas o negativas.

Para la estadística multivariada, se realizó un análisis de asociación entre las variables ambientales y económicas de Costa Rica, utilizando una correlación de Pearson. Luego se realizó un análisis de regresión múltiple donde se consideró como variable dependiente la emisión total de gases de efecto invernadero (TGEI) y como variables independientes (predictoras) las variables consumo de energía en kilogramos equivalentes de petróleo, consumo de energía en kWh per cápita, PIB en dólares per cápita e inversión directa extranjera por año en dólares. Se realizó análisis de regresión logística ajustada por Bonferroni, con modelo de entrada de datos paso a paso o stepwise, donde se consideró una variable predictora significativa si $\mathrm{p}<0,05$. Para realizar este análisis, se usó el paquete estadístico SPSS v21.

\section{Alcances y limitaciones del estudio}

Al tomar datos de una gran cantidad de años y contrastar dos bases de datos abiertas, la metodología permite visualizar tendencias sólidas que no se ven afectadas por un evento fenomenológico puntual; esto permite obtener un modelo metodológico multivariado que brinda solidez a los resultados para los años 1990 a 2014.

Una limitación de este estudio es la necesidad de ampliarlo al usar indicadores sociales y otros elementos que nos permitirían vincular si las emisiones de GEI, así como el PIB, ingresos por turismo, inversión dirigida a fondos financieros verdes y otros indicadores, pueden vinculares con mejoras en la calidad de vida de la población costarricense. De esta forma, se podría conocer si el tercer elemento de un desarrollo sostenible, el ámbito social, se ha visto beneficiado por el crecimiento PIB, pues este estudio solo exploró el ambiental y el económico, pues no tiene pretensión de incluir el eje social.

\section{RESULTADOS Y DISCUSIÓN}

Fueron seleccionados varios indicadores económicos, de consumo energético y de gestión ambiental para tener un panorama de la situación de Costa Rica desde 1990 hasta 2014 (tabla 1). Costa Rica muestra tendencias positivas en el incremento anual de los indicadores financieros de PIB per cápita, ingreso bruto nacional e inversión extranjera (figura 1).

La información obtenida de las bases de datos abiertas del Bando Mundial y del CIAT arrojan tendencias de mejora en PIB, inversión extranjera e ingreso nacional bruto; pero, como se observa en la figura 2, la tendencia a la producción de GEI en forma total no ha disminuido en los últimos quince años y las líneas de tendencia (pendientes y R $2=$ 0,91) muestran de manera consistente que estas no proyectan disminución. Del grupo de ecuaciones y tendencias, la variable de menor crecimiento fue el consumo de energía equivalente derivada de petróleo (R2 = 0,8). Todas las ecuaciones presentaron pendientes positivas en su graficas de mejor ajuste y todas se ajustaron a modelos lineales, 
con valores de R2 altos. Es interesante notar que el índice la emisión de GEI no presenta ninguna disminución en su tendencia de crecimiento durante el período analizado y se visualiza un crecimiento fuerte del PIB entre 2010 y 2014.

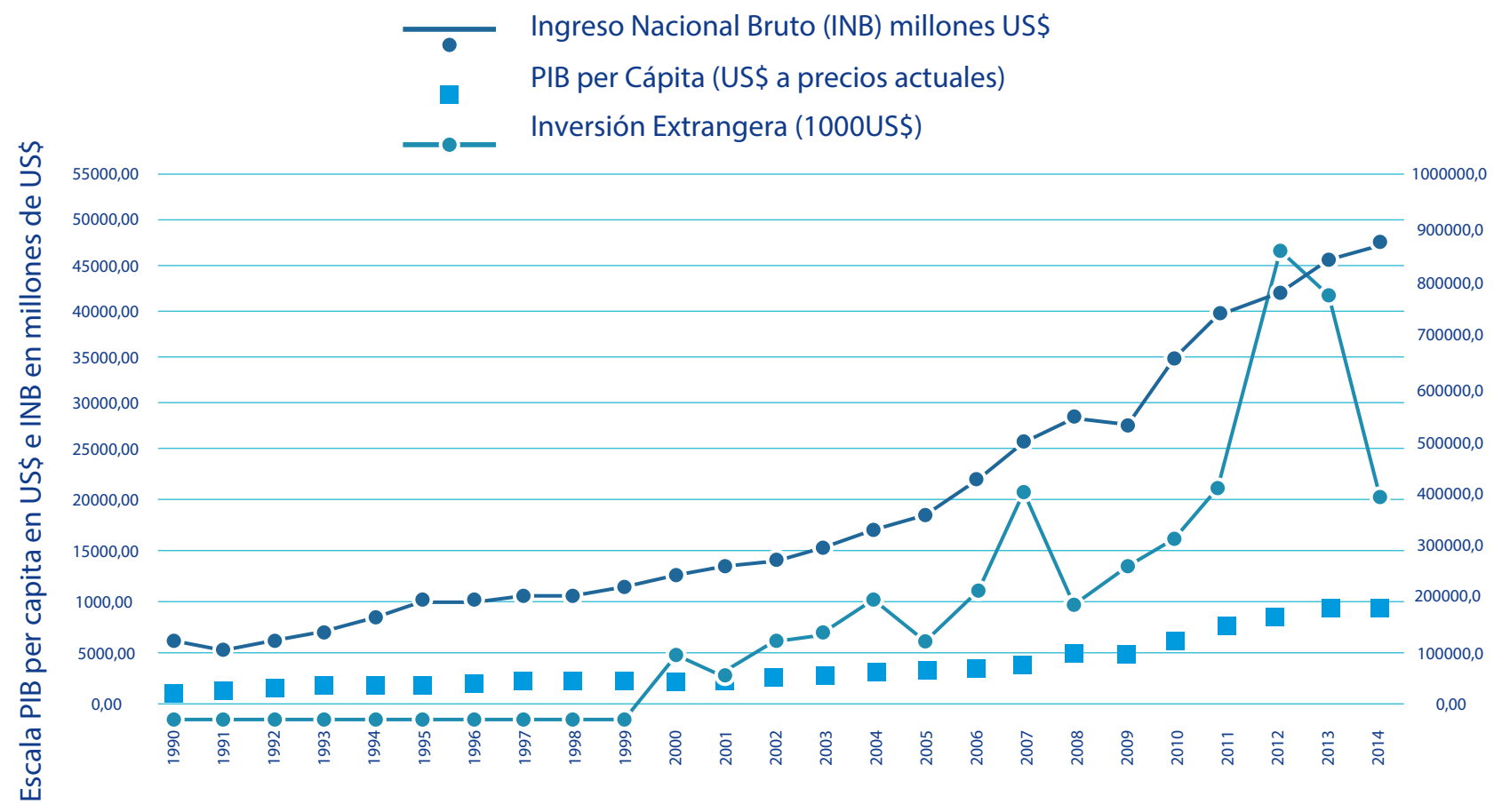

Figura 1. Tendencia de los indicadores económicos PIB, ingreso nacional bruto e inversión extranjera directa desde 1994 hasta el año 2014. Fuente: elaboración propia, con base datos del Banco Mundial (2017).

INB: ingreso nacional bruto, expresado en millones de dólares.

PIB per cápita: PIB per cápita en dólares actuales.

INV. Extranjera: inversión neta extranjera en miles de dólares. 

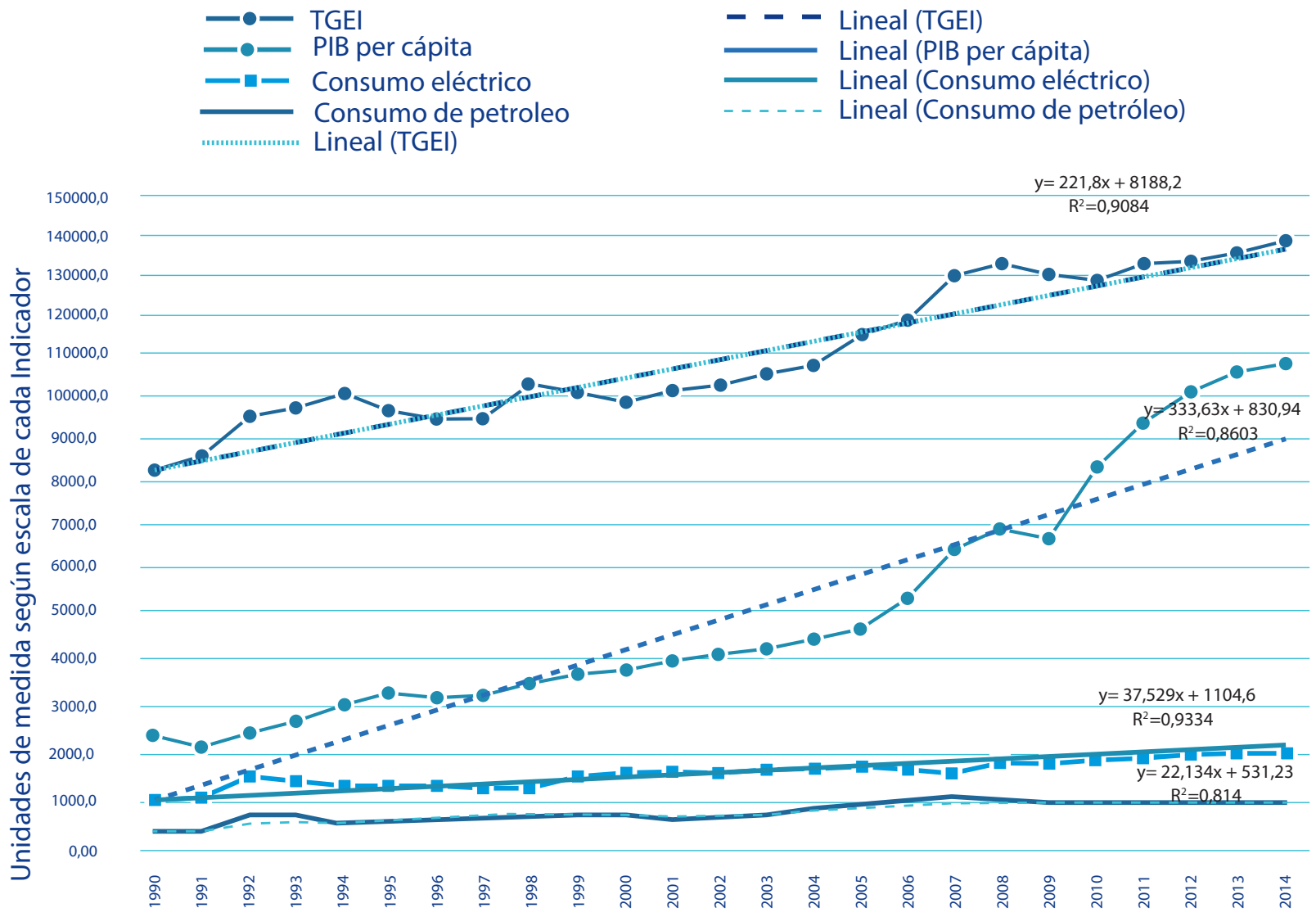

Figura 2. Incremento de los indicadores totales de GEI, PIB, consumo de energía eléctrica y consumo de petróleo equivalente en Costa Rica de 1990 a 2014. Fuente: elaboración propia, con dados del Banco Mundial (World Resources Institute, 2017).

PIB per cápita: dólares a precios actuales.

TGEI: Las emisiones totales de GEI se expresan en Kton.

Consumo eléctrico per cápita: KWh.

Consumo de petróleo: kg equivalente de petróleo.

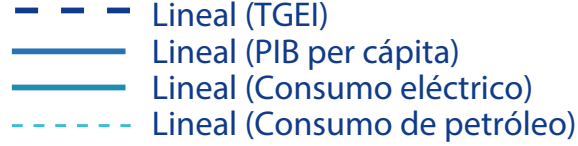

$y=221,8 x+8188$ 
Como siguiente etapa, se determinó que todas estas variables están asociadas y poseen correlaciones de Pearson positivas y significativas con $\mathrm{p}<0,01$. Los valores de las correlaciones son superiores en todos los casos a 0,79 y se observa que el PIB per cápita está correlacionado en un 0,94 con la emisión total de GEI (tabla 2). Estos análisis nos indican que el crecimiento económico de Costa Rica y los procesos de usos de los fondos procedentes de inversión extranjera, así como el uso de la energía emisora de GEI, están correlacionados. Las pendientes positivas halladas en las ecuaciones de mejor ajuste mostradas en la figura 1, concuerdan con los hallazgos de las correlaciones presentadas en la tabla 2.

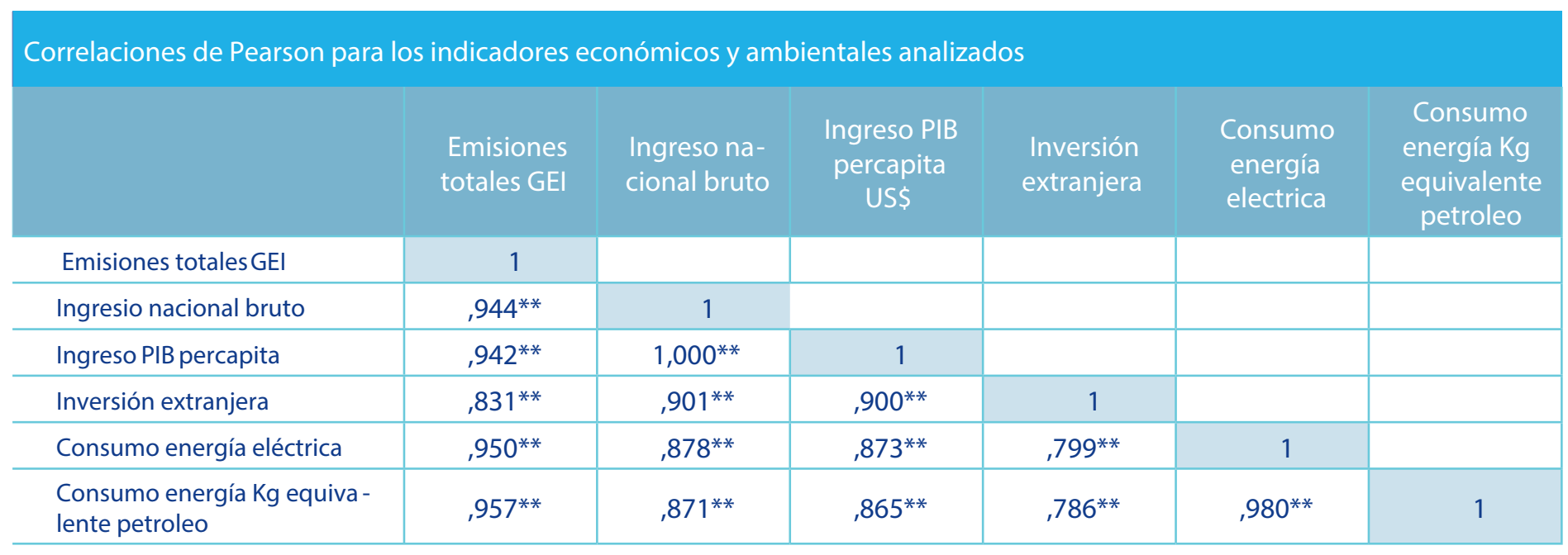

Tabla 2. Correlaciones de Pearson para los indicadores económicos y ambientales analizados Fuente: Elaboración propia.

**Nota: La correlación de Pearson es significante a 0,O1 (2-colas). Los indicadores son emisiones totales de GEI en Kg ton equivalentes de $\mathrm{CO} 2$, sin incluir emisiones de cambio de uso de suelos y plantaciones forestales, los indicadores económicos son el Ingreso Nacional Bruto (millones de dólares), el ingreso PIB per cápita en dólares actuales, la inversión extranjera directa (miles de dólares), el consumo de energía eléctrica en KWh per cápita y el consumo de energía en kg equivalente de petróleo. Fuente: elaboración propia obtenida con datos del Banco Mundial (World Resources Institute, 2017). 
Una vez determinado que todos los indicadores del estudio correlacionan positivamente, se debe analizar cuáles tienen mayor predicción de manera significativa para la emisión de GEI y pueden explicar la variación de estos indicadores Para esto, se realizó un análisis de regresión logística bajo la construcción de modelo de ecuación paso a paso o stepwise, tomando como variable dependiente la emisión total de GEI. El modelo detectó que las variables predictoras fueron el PIB per cápita y el consumo de energía derivada de equivalentes por kilogramo de petróleo. Bajo este modelo, el $96 \%$ de los cambios eran predecibles por estas variables, donde $\mathrm{F}[1,22]=35,16$, con $\mathrm{p}<0,01$ y la R2 ajustada a 0,965 (tablas 3 y 4). Las variables inversión neta extranjera e ingreso nacional bruto fueron excluidas del modelo. La variable consumo eléctrico per cápita en KWh fue utilizada por rigurosidad metodológica y no fue significativa $(\mathrm{p}>0,05)$, aunque, si hubiera existido una asociación estadística, esta sería por azar, pues en los últimos años Costa Rica genera al menos un 98 \% de su energía eléctrica con fuentes renovables y se han dado períodos de más de 250 días sin usar hidrocarburos para generar electricidad.

\begin{tabular}{|c|c|c|c|c|c|c|c|c|c|}
\hline \multicolumn{10}{|c|}{ Resultados del análisis de regresión con las variables del estudio } \\
\hline \multirow[b]{2}{*}{ Modelo } & \multirow[b]{2}{*}{$\mathrm{R}$} & \multirow[b]{2}{*}{$\mathrm{R}^{2}$} & \multirow{2}{*}{$\begin{array}{c}\mathrm{R}^{2} \\
\text { Ajustada }\end{array}$} & \multirow{2}{*}{$\begin{array}{l}\text { Error Std de } \\
\text { la estimación }\end{array}$} & \multicolumn{5}{|c|}{ Estadísticos } \\
\hline & & & & & $\begin{array}{c}\text { Cambio en } \\
\mathrm{R}^{2}\end{array}$ & F Cambio & df1 & df2 & $\begin{array}{l}\text { Sig. F } \\
\text { Cambic }\end{array}$ \\
\hline 1 & $957^{a}$ & ,916 & ,913 & ,5058164 & ,916 & 252,163 & 1 & 23 &, 000 \\
\hline 2 & 984 b & ,968 & ,965 & 3208377 & ,051 & 32,167 & 1 & 22 &, 000 \\
\hline \multicolumn{10}{|c|}{ a. Predictoras: (Constante), CONSUMO ENERGIA KG EQUIVALENTES PETROLEO PER CAPITA } \\
\hline \multicolumn{10}{|c|}{ b. Predictoras: (Constante), CONSUMO ENERGIA KG EQUIVALENTES PETROLEO PER CAPITA, INGRESO PIB PERCAPITA US\$ ACTUALES } \\
\hline \multicolumn{10}{|c|}{ c. Variable Dependiente: TOTAL EMISIONES GEI EN KGTgTon } \\
\hline
\end{tabular}

Tabla 3. Resultados del análisis de regresión con las variables del estudio Fuente: Elaboración propia.

Nota: El modelo tiene de variable dependiente emisiones totales de gases de efecto invernadero (TGEI) y como variables independientes (predictoras) ingreso bruto nacional en miles de dólares, PIB per cápita en dólares, inversión extranjera en miles de dólares, consumo de energía eléctrica per cápita en KWh y consumo de energía en kg equivalentes de petróleo per cápita. Fuente: elaboración propia obtenida con datos del Banco Mundial (World Resources Institute, 2017). 
Resultados del modelo de regresión y la ecuación resultante.

\section{Coeficientes}

Coeficiente

sin estandarizar

Coeficientes

Modelo

B Erroor Std

estandarizados

$\mathrm{T}$

Sig.

Beta

\begin{tabular}{|c|c|c|c|c|c|c|}
\hline 1 & $\begin{array}{l}\text { (Constante) } \\
\text { CONSUMO ENERGIA KG EQUIVA } \\
\text { LENTES } \\
\text { PETROLEO PER CAPITA }\end{array}$ & $\begin{array}{r}3,332 \\
, 009\end{array}$ & $\begin{array}{l}\text {,498 } \\
\text {,001 }\end{array}$ & 957, & $\begin{array}{r}6.695 \\
15,880\end{array}$ & $\begin{array}{l}, 000 \\
, 000\end{array}$ \\
\hline 2 & $\begin{array}{l}\text { (Constante) } \\
\text { CONSUMO ENERGIA KG EQUIVA - } \\
\text { LENTES } \\
\text { PETROLEO PER CAPITA } \\
\text { INGRESO PIB PERCAPITA US\$ ACTUALES }\end{array}$ & $\begin{array}{l}4,984 \\
, 006 \\
, 000\end{array}$ & ,968 & $\begin{array}{l}, 965 \\
, 452\end{array}$ & 3208377 & $\begin{array}{l}, 000 \\
, 000\end{array}$ \\
\hline
\end{tabular}

a. Variable dependiente: TOTAL EMISIONES GEI Kgton

Tabla 4. Resultados del modelo de regresión y la ecuación resultante

Fuente: Elaboración propia obtenida con datos del (World Bank, 2017) ; (World Resources Institute, 2017)

a. Variable dependiente: TOTAL EMISIONES GEI KgTon

Fuente: elaboración propia obtenida con datos del Banco Mundial (World Resources Institute, 2017).

Los resultados de la tabla 4 presentan que la constante de la ecuación es 4,984; la variable independiente de consumo de energía en $\mathrm{kg}$ equivalentes $(P)$ tiene una beta estandarizada de 0,566 y la variable independiente ingreso PIB per cápita (PIBC) en dólares a precios actuales tiene un beta estandarizado de 0,452. Se obtiene entonces la siguiente ecuación final:

$$
\text { GTEI (gases totales efecto invernadero) }=0,566 P+0,452(P I B C)+4,984
$$

La regresión multivariada de GTEI tiene como variables predictoras positivas el consumo de energía equivalente en kilogramos de petróleo y el ingreso bruto per cápita en dólares, donde $F[1,22]=35,16$, con $p<0,01$ y la R2 ajustada a 0,965. Este resultado, con un valor de $F$ alto y $R$ elevado, permite indicar que el 96,5\% del modelo es explicado por estas dos variables independientes (predictoras), con una confianza del $99 \%(p<0,01)$. 
El crecimiento del PIB per cápita en dólares y el consumo de energía en kilogramos equivalentes de petróleo predicen un aumento de las emisiones totales de GEI $(\mathrm{R} 2=0,96)$. Este dato es mucho mayor que los hallazgos encontrados en un estudio realizado en países de la Unión Europea, donde las emisiones netas de $\mathrm{CO} 2$ como variable independiente mostraron un $\mathrm{R}_{2}=0,60$ con la variable predictora PIB (Draghicescu, 2010). Aunque ambos modelos no son comparables en toda su forma, sí nos demuestran que el PIB, tanto en países de la Unión Europea como de Costa Rica, están asociados con la emisión de GEI, tal como lo plantea el estudio de la OECD (2017).

Esta tendencia ha sido también observada en países como Bangladesh, donde las emisiones de CO2 son asociadas con el crecimiento económico (Zakir-Saadullah, 2013). Lo cual nos hace suponer que un CV requiere gran diálogo y metas en todos los sectores para que los sectores público y privado puedan detener las emisiones de GEI sin mermar el crecimiento del PIB.

Los resultados obtenidos para este período de tiempo analizado no difieren de la gran cantidad de estudios publicados a nivel mundial en diversas naciones. Los resultados, en forma general, han mostrado una tendencia bidireccional; consumo de energía y crecimiento económico se asocian positivamente y, si la energía cesa de consumirse, el crecimiento disminuye, de tal forma que la gestión y desarrollo de estrategias de crecimiento donde las fuentes de energía sean libres de emisiones de GEI es imperativa. Algunas naciones desarrolladas han logrado desacoplarse de este modelo y han logrado crecer sin afectar el medio ambiente (Mardani, Streimikiene, Cavallaro, Loganathan y Khoshnoudi, 2019).

Costa Rica pretende lograr cumplir los ODS y el Acuerdo de París para el cambio climático; además, tiene como meta alcanzar la carbono neutralidad. Ante este panorama retador para el país, se visualiza un gran esfuerzo para lograr mejoras ambientales sostenibles, sin afectar la economía, y promover un crecimiento social, como el plan de desarrollo del Minae y otras acciones que se realizan en el país por los pagos por servicios ambientales (Sánchez y Navarrete, $2017)$.

Los resultados obtenidos muestran que durante muchos años ha habido un fuerte acoplamiento del PIB y el consumo de petróleo a las emisiones totales de GEI. Las pendientes mostradas son positivas y sus ecuaciones son positivas; pero, si se observa el período que comprende 2010 a 2014, las gráficas del PIB y el total de emisiones de GEI muestran un desacoplamiento (figura 3). En la gráfica de la figura 3, al usar únicamente los años de 2008 a 2014 , la correlación Pearson es de 0,84 y no de 0,94 para estas mismas variables cuando se analizó todo el conjunto de años. Existe, pues, un elemento a notar: en 2008, Costa Rica declara su intención de ser carbono neutral, ya han pasado más de diez años del arranque de los pagos por servicios ambientales y el país muestra para este período una mayor conciencia ambiental, tanto en sus estrategias país como en la empresa privada. Pero aún estos datos no pueden ser tomados como concluyentes del inicio de un desacoplamiento, pues se requiere analizar el país con los resultados del próximo inventario de gases de efecto invernadero, que está en proceso de conclusión por el Instituto Meteorológico Nacional. Se debe analizar y proyectar desde 2008 hasta 2018, para validar si este proceso ha sido sostenible y si disminuye el poder de las asociaciones estadísticas halladas en este estudio, lo cual sería el evento deseable. 
- - Total de emisiones GEl en kgton

- - PIB per cápita en US\$

_ Lineal (Total de emisiones GEl en kgton)

- - - Lineal (PIB per cápita en US\$)

$16.0000,0$

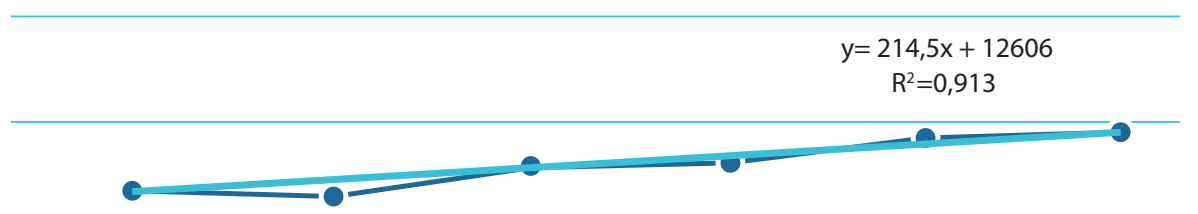

$12.0000,0$

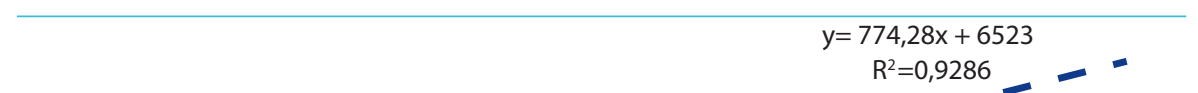

$10.0000,0$

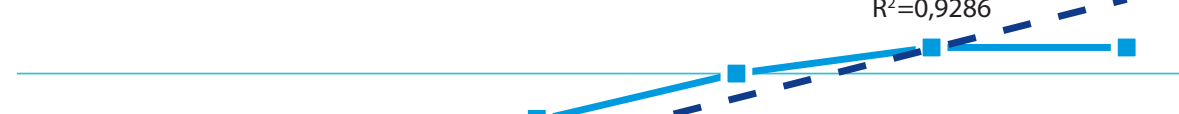

$8.000,0$

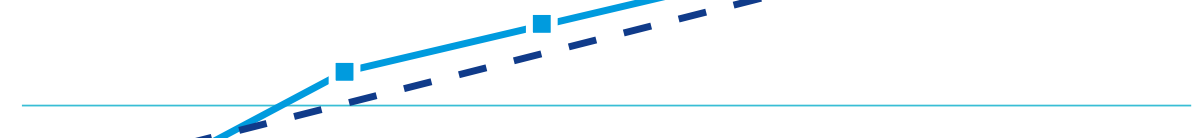

$6.000,0$

$4.000,0$

$2.000,0$

0,00

2009

2010

2011

2012

2013

2014

Figura 3. Emisiones totales de GEl y PIB per cápita para los años 2008-2014. Fuente: elaboración propia obtenida con dados del Banco Mundial (2017).

PIB per cápita: dólares a precios actuales.

TGEI: emisiones totales de GEI, se expresan en Kton. 
Al tomar estos resultados, podemos ver cómo Costa Rica parece estar en ruta hacia un posible desacoplamiento del PIB de las emisiones de GEI. Para proseguir con los análisis, se debe discutir el Índice de Desempeño Ambiental (EPI, por sus siglas en inglés) (Yale Center for Enviromental Law \& Policiy, 2014), donde Costa Rica ocupa la posición 54, superado por países latinoamericanos como Chile y Ecuador, ubicados en las posiciones 29 y 53 respectivamente. En el índice EPI se destaca que Costa Rica muestra incrementos en su rendimiento sobre años anteriores. En el Índice Global de Economía Verde (GGEI), Costa Rica ocupa la honrosa posición número 11, superado únicamente por Brasil y Zambia como países en vías de desarrollo; los otros países ubicados en los primeros diez lugares son de la Unión Europea (Dual Citizen LLC, 2016).

El hecho de que Costa Rica se ubique en una posición tan elevada como la 11 en el índice GGEI en el año 2016 y prácticamente solo superado por países con enormes recursos económicos o naturales, podría inducir a pensar que Costa Rica debería enfocar su norte económico hacia otros problemas como infraestructura, salud, delincuencia, corrupción e incremento en el déficit fiscal. Sin embargo, al analizar el resultado de la figura 1 y los datos estadísticos, se percibe que no se ha logrado contener las emisiones de GEI y el PIB, como indicador de crecimiento, es una variable predictora de mayores emisiones. Los análisis muestran que la combinación del crecimiento del PIB con el aumento en la demanda del consumo de kilogramos equivalentes de petróleo predice un $96 \%$ del modelo de emisiones. Pero, como ya también se analizó, podría existir un posible cambio en esta tendencia (figura 3); lo cual nos hace pensar que Costa Rica está en proceso de superarse en temas ambientales y se posiciona como un país que busca un CV. Estas posiciones se sustentan con los hallazgos para los años analizados en la figura 3, donde se observa un proceso de desacoplamiento parcial y logra coincidir con el estudio de la OECD (2017). Aunque se debe ser consistente en que el comportamiento histórico muestra una fuerte asociación y acoplamiento, el cual debe ser monitoreado en los años 2014 a 2018 para considerar si este proceso es sostenible.

El Gobierno actual (2018-2022) ha lanzado un plan para descarbonizar la economía, donde destaca fuertes elementos para contener la emisión de GEI (MEIC, 2018). Un posible plan de acción sería analizar potenciales reducciones en temas como generación eléctrica por medio de celdas fotovoltaicas (para eliminar toda dependencia de hidrocarburos) y el uso de biocombustibles, aplicaciones en biomasa y potenciar el uso de vehículos híbridos o eléctricos, como potenciales cambios para migrar hacia el CV, la carbono neutralidad y desacoplar el PIB de la emisión de GEI. Para dar soporte a estos proyectos, un estudio reciente demostró, con modelos matemáticos en países de la Unión Europea, que todos los tipos de energía renovable favorecieron al PIB; pero, de todos ellos, la biomasa es la de mayor influencia en el crecimiento económico (Armeanu, Vintala y Gherghina, 2017). Lo cual va en el norte de posicionarse como un país comprometido para contener el cambio climático y lograr los acuerdos adquiridos.

Aun así, el entorno actual de la economía, con un alto desempleo que supera el 13 \% y una sociedad que urge de un plan para la reactivación económica, pueden inducir a pensar que un proceso de descarbonización es costoso y no adecuado para nuestro entorno y podría generar un proceso negativo en el crecimiento económico. Pero, dado que la matriz eléctrica costarricense es con base en fuentes renovables, el control de emisiones sobre fuentes derivadas de petróleo está más asociado al transporte (Chacón, Jiménez, Montenegro, Sasa y Blanco, 2012), lo cual indica a los tomadores de decisiones de políticas públicas cuáles ejes del plan de descarbonización pueden ser atacados rápidamente, sin afectar el crecimiento de la economía. 


\section{CONCLUSIONES}

Los datos obtenidos muestran que la emisión de gases de efecto invernadero (GEI) es predecible por los indicadores económicos PIB per cápita en dólares y el consumo en kilogramos equivalentes de hidrocarburos, según el período de análisis de 1990 a 2014. Estos datos, para un país con fuerte tendencia a proponer una imagen verde, obligan a considerar nuestro actual formato económico y explorar dónde se puede desacoplar nuestro modelo de crecimiento de las emisiones de GEI y así lograr un crecimiento verde (CV) sostenible. Los datos disponibles al último inventario nacional de GEI en 2012 hacen imposible valorar si el comportamiento que se da entre los años 2008 y 2014 son sostenibles hacia el 2020. El elemento valioso de este estudio es que muestra los años posteriores al 2008, donde parece comenzar a emerger una tendencia diferente y se observa un posible desacoplamiento. Por lo tanto, se demostró que el crecimiento económico históricamente estuvo acoplado a las emisiones de GEI, pero es necesario realizar un estudio más reciente cuando se disponga de los datos de un nuevo inventario nacional de GEI para determinar si esta tendencia es continua y si es un limitante para llegar a un crecimiento verde. La hipótesis del estudio que esperaba un desacoplamiento fuerte de las emisiones de gases de efecto invernadero, luego de las políticas y estrategias verdes empleadas por Costa Rica, no logra comprobarse. Se debe considerar que la hipótesis para estos años debe ser rechazada y vincular el crecimiento del PIB a las emisiones de GEI, como ha ocurrido y ocurre en muchas naciones. El nuevo inventario de GEI nacional que promueve el Instituto Meteorológico Nacional de Costa Rica es vital para poder valorar si Costa Rica está o no en rumbo a un crecimiento verde, pues se observa una posible tendencia diferente entre los años 2011 y 2012, pero el período es reducido para poder llegar a conclusiones sólidas. 


\section{BIBLIOGRAFÍA}

Allen, C. y Clouth, S. (1 de 08 de 2012). A guide to green economy (UNDESO ED). Recuperado de https:// sustainabledevelopment.un.org/index.php?page $=$ view\&type $=400 \& n r=634$

Armeanu, D., Vintala, G. y Gherghina, S. (2017). Does renewable energy drive sustainable economic growth? Multivariate panel data evidence for EU-28 countries. Energies, 10, 381-402.

Boslaugh, S. (2013). Statistic, In a nutshell. A desktop quick reference guide (2da. ed.). Sebastopol: O'Reilly Media, Inc.

Chacón, A., Jiménez, G., Montenegro, J., Sasa, J. \& Blanco, K. (2012). Inventario nacional de gases efecto invernadero y absorción de carbono 2012. San Jose: Minae, IMN, GEF, PNUD.

Comisión Europea - Acción por el Clima. (Diciembre de 2015). Acuerdo de París. Recuperado de https://ec.europa.eu/ clima/policies/international/negotiations/paris_es

Draghicescu, M. (1 de enero de 2010). Study of correlation between the gross domestic product and the greenhouse gas emissions throughout the European Union by using econometric models. 5th International Conference on Applied Statistics, (págs. 1-12), Bucharest. Recuperado de http://search.ebscohost.com/login.aspx?direct=true\&site=edslive\&db=asn\&AN=64925019

Dual Citizen LLC. (2016). GGEI: Measuring national performance in the green economy. New York: Dual Citizen LLC.

GGGI. (2015). Accelerating the transition to a new model of growth-GGGI refreshed stratetic plan 2015-2020. Recuperado de http://gggi.org

Greiner, A. (2004). Global warming is a basic endogenous growth model. Evinronmental Economics and Policy Studies, 6, 49-73.

Mardani, A., Streimikiene, D., Cavallaro, F., Loganathan, N. y Khoshnoudi, M. (2019). Carbon dioxide (CO2) emissions and economic growth: A sistematic review of two decades of research from 1995 to 2017. Science of the Total Environment, 649, 31-49.

Martínez, A. y Porcelli, A. (2017). Reflexiones sobre la economía verde. El new deal ecológico mundial. Lex, 15(19), 361-408.

MEIC. (2018). Plan de Descarbonización. Compromiso del Gobierno del Bicentenario 2018-2022. Recuperado de www.minae.go.cr

Millenium Ecosystem Assestent. (1 de marzo de 2005). Living beyound our means. (W. Reid, ed.) Recuperado de: http://www.wri.org/publication/millennium-ecosystem-assessment-living-beyond-our-means

Minae. (5 de diciembre de 2017). Propuesta de política nacional de consumo y producción sostenible 2018-2030. Recuperado de: http://www.digeca.go.cr/documentos/propuesta-de-politica-nacional-de-consumo-y-produccionsostenibles

Minae. (2018). Plan Nacional de Descarbonización. Compromiso del Gobierno del Bicentenario 2018-2022. Recuperado de: https://minae.go.cr/images/pdf/Plan-de-Descarbonizacion-1.pdf

Moore, F. y Diaz, D. (2015). Temperature impacts on economic growth warrant stingent mitigation policy. Nature Climate Change, 5, 127-131.

OECD.(11 demayode2011). Toward greengrowtheconomy.Recuperadode:http://dx.doi.org/10.1787/9789264111318en

OECD. (2017). Green Growth Indicators. P. OECD Publishing. doi: http://dx.doi.org/10.1787/9789264268586-en 
Organización de las Naciones Unidas. (25 de setiembre de 2015). Objetivos para el desarrollo sostenible. Recuperado de: http://www.un.org/sustainabledevelopment/es/objetivos-de-desarrollo-sostenible/

Sánchez, O. y Navarrete, C. (2017). La experiencia de Costa Rica en el pago de servicios ambientales: 20 años de lecciones aprendidas. Revista de Ciencias Ambientales, 51, 195-214.

Banco Mundial (20 de noviembre de 2017). World Bank’s Open Data. Recuperado de https://data.worldbank.org/ data-catalog

World Resources Institute. (2017). CAIT Climate Data Explorer. Recuperado de http://www.cait.wri.org

Yale Center for Enviromental Law \& Policiy. (2014). Envriomental Perfomance Index. Universidad de Yale. Recuperado de http://archive.epi.yale.edu/content/2014-epi-report

Zakir-Saadullah, M. (2013). Causality between economic growth, energy consumption and green house gas emissions in Bangladesh: a Toda-Yamamoto approach. Journal of Academic Research in Economics, 5, 245-257. 